Difficulties and Approaches in the Development of Inventory Control for Nuclear Material Safeguards

S. C. Suda

Oetober 20, 1970

Prepared for the 38th National Meeting of the Operations Research Society of Americe. Detroit, Michigan October 27-30, 1970

LEGAL NOTICE

sponsored was prepared as an account of work sponsored by the United States Government. Neither the United States nor the United States Atomic Energy Commission, nor any of their employees, nor any of their contractors, subcontractors, or their employees, mukes any warranty, express or implied, or assumes any legal liability or responsibility for the accuracy, any pleteness or usefulness of any information, gpparatus, product or process disclosed, or represents that its use would nor infringe privately owned rights. 
Difficulties and Approaches in the Development of Inventory Control for Nuclear Material Safeguards

s. C. Suda

The general problem of inventory control in nuclear materiai safeguards involves decisions concerning the determination of a plant's nuclear materials inventory. The goal of domestic nuclear safeguards in the United States is to provide suitable assurance that source and special nuclear material existing in the United States and intended for peaceful purposes is not diverted unlawfully to other purposes. It should be noted that inventory verification is just one element of this $n$ dimensional problem matrix.

Obviously, one of the indicators of non-diversion is an inventory which has no shortages or overage. The converse need not be true: apparent shortages of material may be the result of measurement inaccuracies or data handlir.g error.

The objective of inventory verification is a high degree of assurance that the amount and composition of special nuclear material (SNM) declared on inventory by the facility does in tact correspond to the material in itss possession. Eollows:

The decisions concerning inventory verification can be classified as

1. The time and cost for inventory verification are fixed and the measurement method, the number of items to be measured, the sampling method and the degree of conformance required of each item measured with its declared value must be determined.

2. In addition to the above, the inspection level for each class of strategic value of material is to be determined and the frequency of inventory verification is to be determined as a fulction of the strategic value.

For the purposes of this report, material of high strategic value is defined to be uranium containing greater than $10 \%$ by weight of $235 U$ and $233 U$, combined, or plutonium of any isotopic composition.

The research problem is to find ways of optimizing such decisions. An optimum decision, in this discussion, j.s one which maximizes the overall level of assurance for a fixed anount of inspection or verification effort.

One of the major difficulties in inventory verification is the determination uf assurarce value for the various verification variables consicared. 'ihere are three types of assurance that are sought:

A. the assurance that there are no missing or empty containers in the inventory

B. the assurance that there is not a large number of containers each with a small amount missing in the inventory

c. the assurance that there are not some containers in the inventory in which the nuclear material has been replaced with a facsimile. 
In addition to the verification variables, two other classes of variables are involved in general inventory verification problems: These are plant variables and regulation variables.

Plant Variables: Plant variables are those variables which are influenced by plant conditions and involve the type of process performed by the plant, the economics of material handling and the management philosophy of the plant. Some facilities may have large inventory holdings of many similar items as in a chemical reprocessing plart, others may have many small dissimilar items as in a fual fabrication plant. Plant variables are:

1. the size and item diversity of the inventory

2. the amount of material of high strategic value on inventory

3. the measurement capability of the plant

4. the proportion of difficult-to-measure material on inventory (This may be due either to material heterogeneity or the form of the material such as sludge or scrap.)

5. the type of plant: Is the material balance for the plant under consideration flow dominated or inventory dominated?

6. the inventory handling and stocking policy of the plant.

Requlation Variables: Federal regulations once promulgated can not be considered variables. However, a regulatory body may from time to time add or withdraw regulations as it deems fit. Therefore, it is meaningful to consider and evaluate various possible regulations which may simplify or add substancially to the effectiveness of certain verification variables. Once identified, the safeguards authority could then consider the possibility of publishing such regulations. Some regulations which might be considered are:

1. an upper limit on the amount of scrap and other hard-to-measure material permitted for a given type of plant

2. an upper limit on the ratio of scrap and other hard-to-measure material to total inventory holding for all plants

3. the degree of physical inventory taking (measurements) required by the plant in preparing for the inspection

4. the form and format the plant shall use in preparing its listing of the inventory

5. segregation of the active inventory items from the inactive and the use of seals to hold fixed the inactive inventory

6. either full or stepwise cleanout of process streams. In a stepwise cleanout, each subprocess area, in turn, is isolated, depleted of material and removals and remaining holdup are measured.

In this study, the cost of performing the : Jentory is a control variable. other control variables are measurement capability and the regulation variables.

Nuclear Materials Accountability: Facilities which possess and process special nuclear materials are responsible for the "accountability" of these 
materials. Nuclear materials accountability involves activities concerning the acquisition, recording, analysis and presentation of quantitative data necessary for the control of the nuclear material held by the facility. The in-process operations which the accountability office is responsible for are the calibration of measurement instruments, tiie measurement of the flow of nuclear materials, and the measurement of the nuclear material in inventory. Functionally, the accountability activities include a measurement and record systems, data analysis and a reporting system. The flow of data and the relationship between the operational and functional activities is shown in Figure 1.

Inspection of Nuclear Facilities: Inventory verification is but one of a number of activities which the inspectors perform as part of the annual inspection of a facility. The process of inventory verification can not be studied independent of the other materials accounting activities. These activities are listed in Table 1 .

\section{Materials Accounting}

\section{Plant Operators' Responsibilities}

1. measurement and calibration methods

2. data flow and data processing

3. records and reports

4. annual inventory

5. analysis of measurement and calibration data

\section{Safeguards organization Activities}

1. test and review measurement system

2. evaluate data system

3. audit of records and reporting syste.n

4. verification of declared inventory

5. review of statistical techniques

Taille 1

In addition to the evaluation of the adequacy of the nuclear materials accounting system, the inspectors also review the effectiveness of plant management's administrative control measures.

The topics covered by the facilities' administrative control measures are facility organization, physical security, accessibility of nuclear materials, shipper-receiver differences and internal safeguards reviews.

Inventory Verification: Inventory verification is usually coupled with the plant's annual inventory required by federal regulations and involves independent measurements by inspectors of a selected number of inventory items. The plant may be viewed as one or more material balance areas and the itens which are to be measured by the inspectors are determined by stratification of the inventory and the use of statistical sampling plans. The inventory may be stratified according to various characteristics. Where the statistical properties of the measurement data are concerned, the stratification usually divides the inventory into groups of material having a common measurement error. The sampling level of the inactive inventory may be different from that of the active inventory. An attempt may or may not be made to independently establish an estimate of the total quantity of SNM on hand at the facility. Because of 
Nuclear Materials Accountability

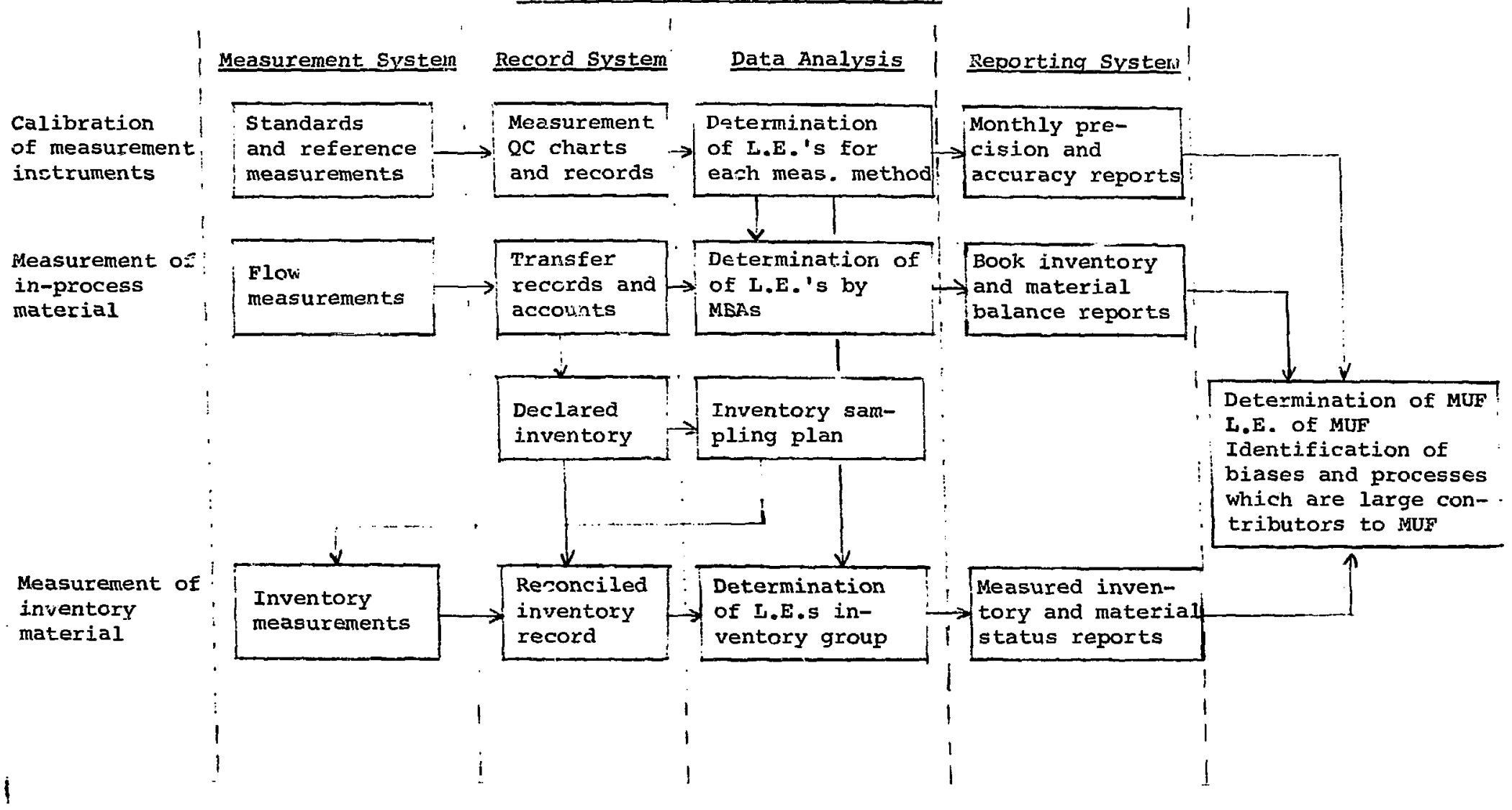

Figure 1 
these and other constraints, the level of statistical confidence associated with the declared inventory is often difficult to ascertain.

The problem of inventory varification is one of specifying the procedures for and the conditions under which sample results can be usea to make unbiased and reasonably precise inferences with respect to the consequences of safeguards decisions.

In order to apply standard statistical sampling plans for inventory verification, the following information is required:

1. the basis of verification (e.g., piece count, item identification, weight check, chemical assay, etc.)

2. the method of describing the conformance of the quality to specified requirements (i.e., the method for classj.fication of defects)

3. the basis for material stratification (what constitutes a verification lot?)

If the objective of inventory verification can be formulated mathematically within the framework of the above, standard statistical methods can be used to specify:

1. the kind of sampling procedure to be employed

2. the kind of sample statistic to be computed from: the verification data, and

3. the logical rules of inference to be used in generaiizing the results of inventory verification.

Further, once the amount of precision desired in the statistical inferences is specified and the measurement methods have been selected, it is possible to determine the minimum sample size.

As noted earlier, the inspection level and frequency of inspection for a class of material is to be determined as a function of its strategic value. The method of inventory verification considered below is applied to material. of low strategic value. For materials of high strategic value, a higher level of assurance is required and a separate approach should perhaps be considered.

Inventory Verification of Low Strategic Value Material: Inventory verification procedures should provide a high probability of detection of loss of special nuclear materials within the time period needed for conversion to weapons use. For material of low strategic value, this conversion would be expensive and time consuning and the quantity of material required to pose a given threat would be relatively large. Thus, the time interval which might be permitted to lapse before positive indicators of material loss are encountered need not be the same as that for material of high strategic value.

Because of cost and other reasoins, it is normally inpossi". 1e to measure the SNM content of every item of low strategic value on inventory. There is a need therefore, for a method of drawing inferences about the inventory based on samples drawn from the inventory. 
Suppose the declared inventory at a facility is $20,000 \mathrm{kilograms}$ of low snriched uranium. Intuitively, the straightforwardness of testing the hypothesis that the total amount of material on inventory at the facility is at least $20,000 \mathrm{kilograms}$ of uranium is very appealing. in practice, it is very difficult to validly make this test. This is because tests of hypotheses, in general, are applicable only to means, variances and proportion: . A partial dodge is the use of lot acceptance sampling plans such as Mil-std 105D and 414 based on inventory lots which have been determined according to some grouping or stratification plan. The general procedure which characterizes lot-by-lot sampling inspection is that items are formed into lots, a sample is drawn from each lot and a particular lot is either accepted or rejected based on one or more quality characteristics of the sample.

The result of this is a lot-by-lot acceptance of the declared inventory. The problem of deciding how many lcts can be rejected and the declared inventory still found acceptable remains. Also there are serious difficulties associated with the use of Mil-std $105 \mathrm{D}$ and 414 in sampling chemical compounds. This is because the concept of percent defective, which is er ressed in terms of AQLs (acceptable quality level) and is basic to these plans, has no meaning in connection with sampling chemicals per se [1]. Except under certain limited conditions and assumptions, Mil-std 105D is not applicable for the sampling of bulk material. Military standards for sampling are designed to estimate average lot quality without $a_{i i_{y}}$ restriction on the variability which may exist within a lot [2].

The principal limitations to the use of - ither attribute or variabls 5 acceptance sampling plans in inventory verification is that they involve the assumption that the process by which a "defective" item is identified is exact (i.e., the measurement error is sufficiently close to zero). In addition lot acceptance sampling by variables involves the assumptior that the distribution of the qualicy variable being measured in the inspection lot is normally distributed. These assumptions are generally inapplicable to many situations of inventory verification.

As an alternative to lot acceptance sampling, the inspection team may choose to examine the arrangement of the inventory verification program to see if the technique of paired observations is applicable. The notable features of this technique are that 1) the variation between the items on inventory does not influence the variance of the mean difference between the results obtained by the plant a.1d the inspector and 2) by inference, a lower (or upper) bound can be calculated for the amount of material in the possession of plant operator.

The Inventory Problem: Let the array (W) represent the items in the declared inventory. From the $\mathrm{N}$ items on inventory, the inspector draws a sample of $n$ items. Let $x_{i}$ represent the declared inventory value for sample $i$ tem $i$ and $y_{i}$ the measurement value obcained by the inspector. The calculated sample statistics are:

$$
\begin{aligned}
\mathrm{d} & =x_{i}-y_{i} \\
\bar{d} & =\Sigma d_{i} / n \\
s_{d} & =\left[\Sigma\left(d_{i}-\bar{d}\right)^{2} /(n-1)\right]^{\frac{1}{2}} \\
s_{m} & =\left[\Sigma d_{i}^{2} / 2 n\right]^{\frac{1}{2}} \\
s_{z} & =\left[s_{x}^{2}+s_{y}\right]^{\frac{1}{2}}
\end{aligned}
$$


The relationship between the trie unknown (metaphysical) values of the items in a storage vault and the declared inventory, sample drawn for inventory. Verification and the computed scatistics are shown in Figure 2.

If the exrors in measurements are independent of the items measured, the corresponding observations are assumed to have the same value apart from random variations. That is, the differences $d_{i}=x_{i}-y_{i}$ all have true value zero. Further, is the measurement errors are normally distributed it is reasonable tc assume, the $d_{i}$ are normally distributed with mean zero and variance $\sigma_{d}{ }^{2}$.

It follows then that the mean difference, $\vec{d}$, is normally distributed with parameters $\left(0, s_{d} / \sqrt{n}\right)$ and that the common ratio $t=\sqrt{n} \bar{d} / s_{d}$ has a $t$ distribution with $n-1$ dearees of freedom.

The $100(1-\alpha) \%$ confidence limits about the parameter $\mu$ is defined by the following expression:

$$
\operatorname{Conf}\left\{\bar{d}-t^{\prime} s \bar{d} \leq u \leq \bar{d}+t^{\prime} \cdot s_{\bar{d}}\right\}=100(1-\alpha) \%
$$

where $t^{\prime}=t(x / 2, d f)$

$$
\begin{aligned}
& t^{\prime \prime}=t(1-\alpha, 2, d f), \text { and } \\
& s_{-}=s_{d} / \sqrt{n}
\end{aligned}
$$

The confidence interval depends upon the sample values $\bar{d}, s$ and $n$. The quantity $t(\alpha / 2)$ is obtained from a table of percentage points of the $t$-distribution with $n-1$ degrees of: freedom. An equivalent of this procedure would be to test whether the average net difference, $\bar{d}$, is significantiy different from zero.

If the errors of measurements are independent of the items measured, then the two computed variances, $\sigma_{\mathrm{m}}{ }^{2}$ and $\sigma_{\mathrm{z}}{ }^{2}$ have the same theoretical variance and the variance ratio

$$
x^{2}=\sigma_{m}^{2} / \sigma_{z}^{2}
$$

has a true value one.

The variance, $\sigma_{m}^{2}$, depends only on the variation of the differences between the measured values and is estimated by [3].

$$
s_{m}^{2}=\Sigma a^{2} / 2 n
$$

The varjance $\sigma_{z_{2}}^{2}$ is obtained by statistically propogating the measurement variances $\sigma^{2} x$ and $\sigma^{2} y$.

The ratio of the computed variances, $r^{2}$, has an $F$-distribution with $n_{m}$ and $\mathrm{n}_{\mathrm{z}}-\mathbf{1}$ degrees of freedom.

The confidence limits for the variance ratio is defined by

$$
\text { Conf }\left\{s_{m}{ }^{2} F_{1} / s_{z}{ }^{2} s r^{2} s s_{m}{ }^{2} F_{2} / s_{z}{ }^{2}\right\}=100(1-\alpha) \%_{2}
$$

where $F_{1}=F\left(\alpha, 2, n_{1}, n_{2}\right)$ represents the percentage points of the F-distribution for $n_{n 1}, n_{z^{-1}}$ degrees of freedom. Similarly $F_{2}=F\left(1-\alpha / 2, n_{2}, n_{1}\right)$. The test hypothesis would be that there is no difference in the population variances, or in other words, that $r^{2}=1$. 


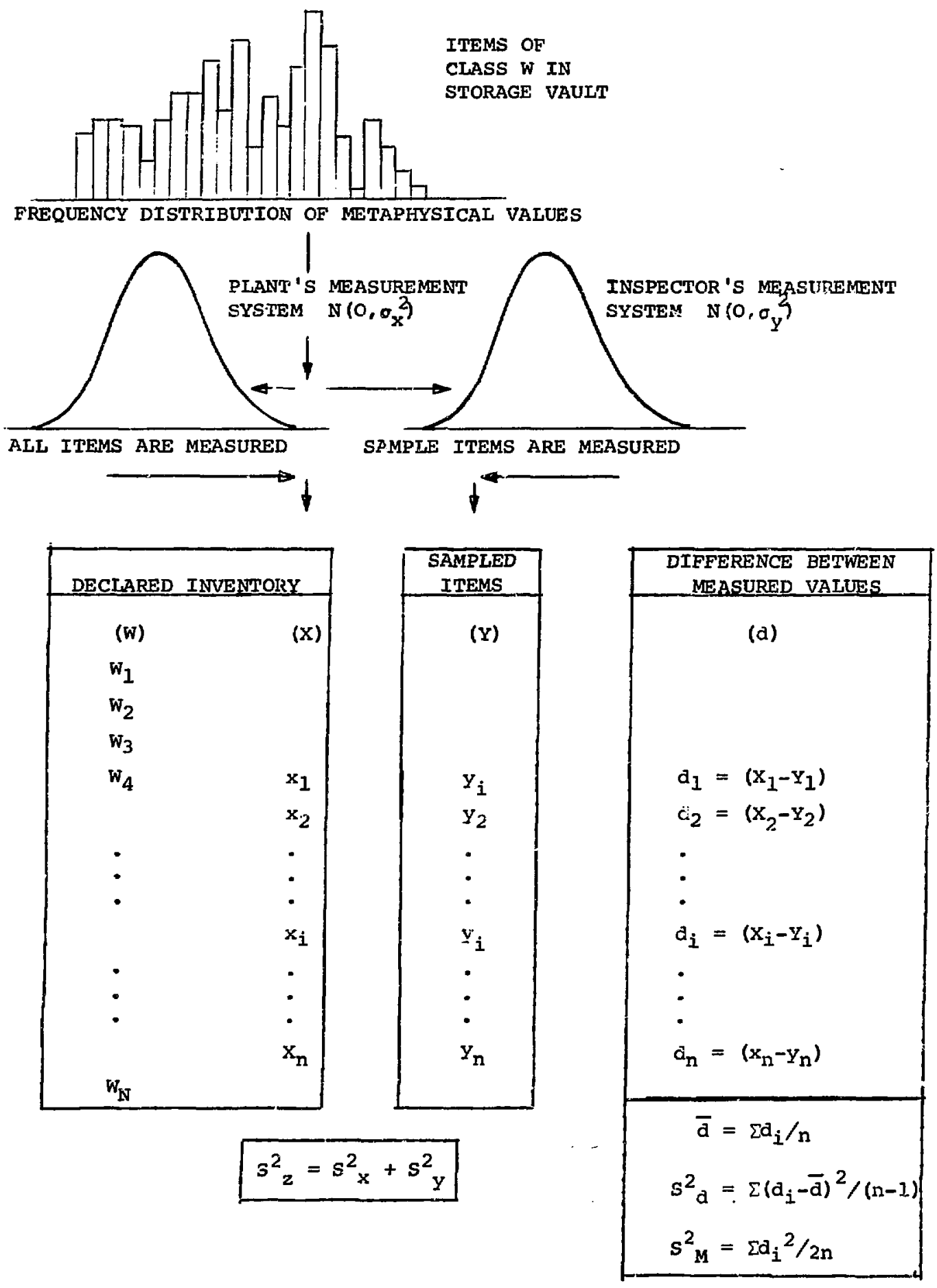


If the information concerning the measurement capability is valid, inferences about the amount of material on inventory may be drawn based on the confidence limits for $\bar{d}$ and $r^{2}$. For a given $\bar{d}$ and lot $w$ consisting of $N$ items, a new estimate for the total amount of material on inventory is given isy $w+i v \cdot \bar{d}$. Additional effort is needed in this area to specify under what conditions one can draw inferences about the quantity $W+N \cdot \bar{d}$ if one or the other of $\bar{d}$ and $r^{2}$ are found to be significantly different from zero and one, respectively. Consideration might also be given to methodology for determining the smallest size of fiducial intervals for $\bar{d}$ which would include the value zero. Similarly for $r^{2}$.

The inspector samples the inventory without replacement. The inventory consists of a number of classes (inventory lots) of in-process or in-storage materials. The total inventory for a given facility will, in general, involve mure than one class of material. The inspection effort to be allotted to a given in-. ventory lot may depend upon:
a) its strategic value (both absolute and relative to the other inventory lots),
b) the total amount of fissionable material involved,
c) its accessibility or ease of losing some part of it,
d) the time lapsed since the last inspection,
e) the "fiducial limits" for the "parent" inventory lot in the last inspection.

The sampling problem is one of defining a plan for stratifying the inventory into viable inventory lcts of assigning fixed costs and fixed effort across a number of inventory lots.

The items selected by the sampling pian may be subjected to bulk measurements, and in turn these items may be sampled for chemical or j.sotopic composition. The fissile content of the entire item may be determined by non-destructive assay. The number of samples to be drawn from a given inventory lot and the type of measurements to be made on the sample item are determined by the samping modeI.

$$
\text { Let } \begin{aligned}
j & =\text { the measurement method } \\
c_{j} & =\text { cost for measurement } j \\
n_{j} & =\text { sample size for measurement } j
\end{aligned}
$$

If the fixed cost for measurements for a given inventory lot is $c$, the cost constraint is expressed $0 ;$

$$
\Sigma c_{j} n_{j} \leqslant c
$$

The Data: An inventory listing for a class of nuclear material is presented in Table 2. The material consists of 150 cans of $3.09 \%$ U-235 enrj.ched uranium in the form of uranium dioxide. Each can contains about 8,010 grams of uranium oxide and the declared amount of $\mathrm{UO}_{2}$ on inventory is 12015 kilograms. The measurea values given in Table 2 are the results of the plint measurements made at the time the material was transferred into the storage vault. 
Inventory Listing: Lot W

\section{A. Item Description}

i. number of items

2. location (sub MBA)

3. type of container

4. item identification

5. iten gross weight ( $g$ )

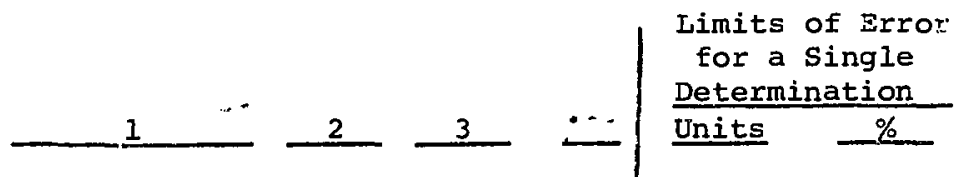

150

storage vault galion can

\section{B. Material Description}

1. type of material $\mathrm{UO}_{2}$

2. physical form powder

3. compound weight (g) 8,017

4. chemical composition (\%)

87.3

5. element weight (g) 6,999

6. isotopic distribution (\% U-235)

3.09

7. isotopic weight (g) 216.3
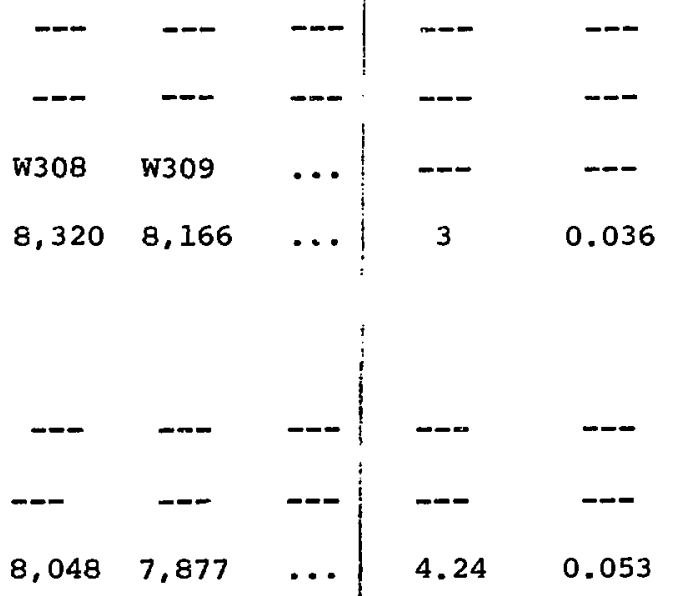

4.24

0.053

n- $\quad \ldots+\ldots$

0.43

0.493

$7,025.6,877$

34.6

0.495

-- --- ---

$0.017 \quad 0.550$

\begin{tabular}{lll|ll}
217.1 & 212.5 & $\ldots$ & 1.60 & 0.741
\end{tabular} 
The limits of error, are two sigma (95\% of nfidence limits) values established by the plant through its calibration an / measurement quality control programs. The units are tiose of the measured values. The base for the percent ITs is the rominal or average value.

Table 3 displays measurement costs and limits of error for various measurement methods available to the inspection team. The sample size and measurement method to be used in measuring the contents of the sampled cans will depend upon the allocated cost for verifying a given class of material and the diversion threat(s) the safeguards authority wants most to protect itself against.

The types of assurance provided by various combinations of the measurement methods are presented in Table 4. The weights of the element and isotope can not be measured directly. These values are derived quantities based on measurements of some functionally-related properties. For example, the weight of uranium is the product of the net weight of the compound and its chemical assay. The measurement sets in Table 4 are defined such that each assurance subgroup is nested within the preceding group.

Applications: Assume the allotted $r$ it for the inspection measurements for lot $\mathrm{W}$ is $\$ 1,500$. We ha. $=$

$$
\begin{aligned}
\Sigma c_{i} N_{i} & =\$ 1,500, \text { and } \\
N & =350 \text { cans. }
\end{aligned}
$$

Several possible measurement strategies are given in Table 5 .

\begin{tabular}{cccccc}
$\begin{array}{c}\text { Measurement } \\
\text { Strategy }\end{array}$ & \multicolumn{5}{c}{$N_{j}$ (costs) } \\
\cline { 2 - 6 } A & $150(1)$ & $18(5)$ & $18(60)$ & $2(90)$ & 5 \\
B & $100(1)$ & $100(5)$ & $15(60)$ & \\
C & $50(1)$ & $50(5)$ & & $48(25)$
\end{tabular}

Table 5

Some hypothetical data have been constructed to test the applicability of methods discussed in this paper. The calculations however are tedious and are better handled by a computer. Etrperical studies, including the writing of a computer code based on these data are planned for the future.

The problems associated with implementation of the above methods are formidable. First, currently, not all plants have the capability to carry out submaterial balance accountability. Second, the application of the plan is possible only if the detail of data routinely reported to the safeguards organization j.s comparable to that listed in Table 2. Third, while the technique of paired observations solves the sampling problem with respect to item-to-item variability, the problem associated with to the sampling for chemical assay of scrap and other difficult-to-measure material remains. 
Table of Measurement Costs

Measurement Method

1. Weighing (1)

2. Weighing (2)

3. Coulometry

4. Mass spectrometry

5. $\gamma$ scanning
Property Measured

gross can weight

net material weight

chemical assay

isotopic distribution

fissile content
Cost/item

$\%$ IE

1

0.03

5

0.05

60

0.61

90

0.16

25

$3 \cdot 5$

Table 3

\section{Measurement Sets}

1. Weighing (gross)

2. Weighing (net)

3. Net weight and coulometry

4. Net weight, coulometry and mass spectrometry

5. Net weight and $\gamma$ sca.rning

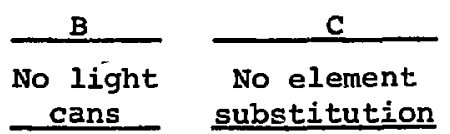

No isotopic No empty cans

$\mathbf{x}$

$\mathbf{x}$

$\mathbf{x}$

$\mathbf{x}$

$\mathbf{x}$

X

$\mathbf{X}$

$x$

$\mathbf{x}$

$\mathrm{X}$

X

$x$

$\mathbf{x}$

X

X 


\section{References}

[1] Mandelson, J. "Chemical Sampling and Classification of Defects," Quality Progress, Vol. 11, No. 11 (Nov. 1969), 8-10.

[2] Rhodes, R. C. "Bulk Sampling," Transactions of the 24th Annual Meeting of the American Society for Quality Control, 1970.

[3] Bennett, C. A., and N. L. Franklin. Statistical Analysis in Chemistry and the Chemical Industry. John Wiley \& Sons Inc., 1954. 